\title{
SYNTHESIS AND CHARACTERIZATION OF SILICON NANOROD ARRAYS FOR SOLAR CELL APPLICATIONS
}

\author{
Brendan M. Kayes, ${ }^{1}$ Joshua M. Spurgeon, ${ }^{1}$ Thomas C. Sadler, ${ }^{2}$ \\ Nathan S. Lewis, ${ }^{1}$ Harry A. Atwater ${ }^{1}$ \\ ${ }^{1}$ California Institute of Technology, Pasadena, CA 91125, USA \\ ${ }^{2}$ University of Cambridge, Cambridge, England
}

\begin{abstract}
Silicon nanorods have been grown by chemical vapor deposition of silane, using both gold and indium as catalysts for the vapor liquid solid (VLS) process. Conditions for optimal rod morphology for each catalyst were identified by varying silane partial pressure and temperature in the range $P=0.05-1$ Torr and $T=300-600 \mathrm{C}$, respectively. In most cases, catalyst particles were formed by partial de-wetting of evaporated films of the catalytic material to form droplets with diameters of tens to hundreds of nanometers. Also, periodic arrays of catalyst particles with controlled size and spacing were achieved both by the use of porous alumina membranes and also by electron-beam lithography. Using these techniques, silicon nanorods were grown with diameters of $100 \mathrm{~nm}$ to microns and lengths of microns to tens of microns. Four-point and gate-bias-dependent resistance measurements were made on single wires, and these indicate that rods we have grown with gold catalysts and phosphine doping have metal-like conductivity.
\end{abstract}

\section{INTRODUCTION}

Nanorods are of potential interest in photovoltaic applications for two principle reasons. First, they allow for decoupling the direction of light absorption from the direction of carrier transport, such that in materials where the diffusion length of excitons or minority carriers is much shorter than the thickness of material required for optimal light absorption, current densities can still be high [1] [2]. Secondly, they allow for the growth of heterostructures of materials with a large mismatch in lattice constant [3], potentially allowing for novel multijunction solar cells. Nanowires can be grown in a variety of different ways, including solution based techniques [4], top-down approaches [5], as well as by introducing silicon from the gas phase (for example in the Vapor Liquid Solid (VLS) process [6]) - note that this is intended neither as an exhaustive list of techniques nor of references.

We have focused our attention on the goal of fabricating silicon ( $\mathrm{Si}$ ) nanorods for wholly inorganic photovoltaic devices based upon pn junctions in either the axial or radial direction. For this reason we are interested in growing rods of a relatively large diameter (at least $100 \mathrm{~nm}$ ), so that the rods can sustain a depletion region, as described in [1]. We use the term "nanorod" rather than the more commonly used "nanowire" to denote this somewhat unusually large size regime. We are interested in growing rods with lengths of tens of ums so that the rod absorbs most of the incident above-bandgap photons. Also, we have focused on the VLS method because we see its potential for rapid, low-cost deposition, scalability, and compatibility with existing $\mathrm{Si}$ thin film deposition techniques as advantageous for our application.

\section{EXPERIMENTAL}

In order to grow Si nanorods by the VLS method, a thermal Chemical Vapor Deposition (CVD) system was used, with silane $\left(\mathrm{SiH}_{4}\right)$ diluted to $5 \%$ concentration in argon (Ar) as the process gas. Metal seed particles were created either lithographically using a lift-off process, or by simple evaporation of a thin $(1-50 \mathrm{~nm}$ ) layer of metal that dewetted from the substrate after deposition to form an array of nanoparticles. We have also explored the use of commercially available porous alumina membranes (Whatman Anopore® inorganic membranes) in combination with the VLS process.

In choosing a catalyst particle to seed nanorod growth, one has to take many factors into account, particularly if one is concerned with making devices [7]. Previously, gold $(\mathrm{Au})$ has primarily been used as the VLS catalyst [6]. However, Au is known to form a deep-level trap state within the band-gap of Si [8], [9]. Although Au has a very low solubility in $\mathrm{Si}\left(\sim 2 \times 10^{-4}\right.$ at. \% [10]), this nonetheless implies that Au concentrations of $\sim 10^{17} \mathrm{~cm}^{-3}$ can be present in nanorods $\left(2 \times 10^{-4}\right.$ at. $\% \times 5 \times 10^{22} \mathrm{Si}$ atoms $\mathrm{cm}^{-3}$ [9]). This can create a high enough trap density to seriously degrade the electronic performance of $\mathrm{Si}$, leading to sub-nanosecond lifetimes [8].

We have explored aluminum ( $\mathrm{Al})$, gallium $(\mathrm{Ga})$, indium (In), and tin (Sn) as potential alternative VLS catalysts. These elements have the advantage over Au of not forming deep-level traps within the band-gap of $\mathrm{Si}$. Of these, we have achieved suitable rod morphologies only with In. Al has the problem of a strong tendency to oxidize and the oxide layer appears to prevent the VLS process [11]. Ga is extremely soluble in Si (segregation coefficient 
0.008 [8]), and rods grown with this catalyst were found to be extremely conical, presumably due to the catalyst particle dissolving as the rods grew. Sn is apparently not thermodynamically favorable as a VLS catalyst for Si [12], and we saw no rod growth with this catalyst.

We have explored variations in pressure, temperature, $\mathrm{SiH}_{4}$ flow rate, and catalyst thickness in order to optimize growth conditions with both $\mathrm{Au}$ and In as catalyst. For the Au catalyst, rod morphology improved with increasing substrate temperature and decreasing $\mathrm{SiH}_{4}$ partial pressure in the range $\mathrm{T}=300-600 \mathrm{C}$ and $\mathrm{SiH}_{4}$ partial pressure $P=0.05-1$ Torr (in agreement with [6]). Best results were achieved with substrate temperatures of 600 $\mathrm{C}$ and $\mathrm{SiH}_{4}$ partial pressure of 0.05 Torr. Flow rate was varied between 40 and $200 \mathrm{sccm}$ but this had relatively little effect on rod morphology, as did thickness of the evaporated Au film.

Results for growth from In catalyst particles are displayed in Fig. 1. Optimal growth was found to occur at lower $\mathrm{T}$ and higher $\mathrm{P}$ for In than for Au. If we exceed substrate temperatures of $500 \mathrm{C}$ we see non-selective growth forming a very rough film. We attribute this to surface diffusion of the In particles upon the Si surface, as well as the high vapor pressure of In relative to $A u$ [13]. If we grow at partial pressures significantly below 1 Torr we see the growth rate drop and we see negligible growth after three hours at partial pressures of 0.1 Torr and less.
Note also that the uniformity of growth across a wafer from untemplated In was extremely poor. The results shown in Fig. 1 are "typical", in some cases in some parts of a given sample we found small regions where the morphology is much better than that shown in Fig. 1, however we have tried to show the most typical morphologies. Fig. 2 summarizes typical optimized growth for In and Au.

\section{Templated Growth}

We have also explored the use of electron-beam lithography and porous alumina membranes to control the catalyst particle size, spacing, and location. In the former case we took <111> p+, $0.01 \mathrm{ohm} \mathrm{cm} \mathrm{Si} \mathrm{wafers} \mathrm{and} \mathrm{an-}$ nealed in oxygen at $900 \mathrm{C}$ for 12 minutes, after a 10 minute RCA clean (1:1:5 hydrogen peroxide:ammonium hydroxide:water at $80 \mathrm{C}$ ) and a 20 minute etch in buffered hydrofluoric acid (BHF). This formed a layer of silicon dioxide $\left(\mathrm{SiO}_{2}\right)$ approximately $10 \mathrm{~nm}$ thick, measured by spectral ellipsometry. The wafers were then spin-coated with a double layer of PMMA, and an electron beam (Hitachi S-4100 FESEM) was used to pattern various 100 um $x 100$ um arrays of circles, with diameter ranging from 100 $\mathrm{nm}$ to $5 \mathrm{um}$, and pitch of either 1 or $10 \mathrm{um}$. After developing the patterns with MIBK and isopropanol (IPA), the oxide was etched in the places from which the PMMA had been removed, by immersion in BHF. Then $10 \mathrm{~nm}$ of catalyst (either $\mathrm{Au}$ or In) was evaporated and acetone used to lift-off the remaining PMMA and unwanted catalyst,

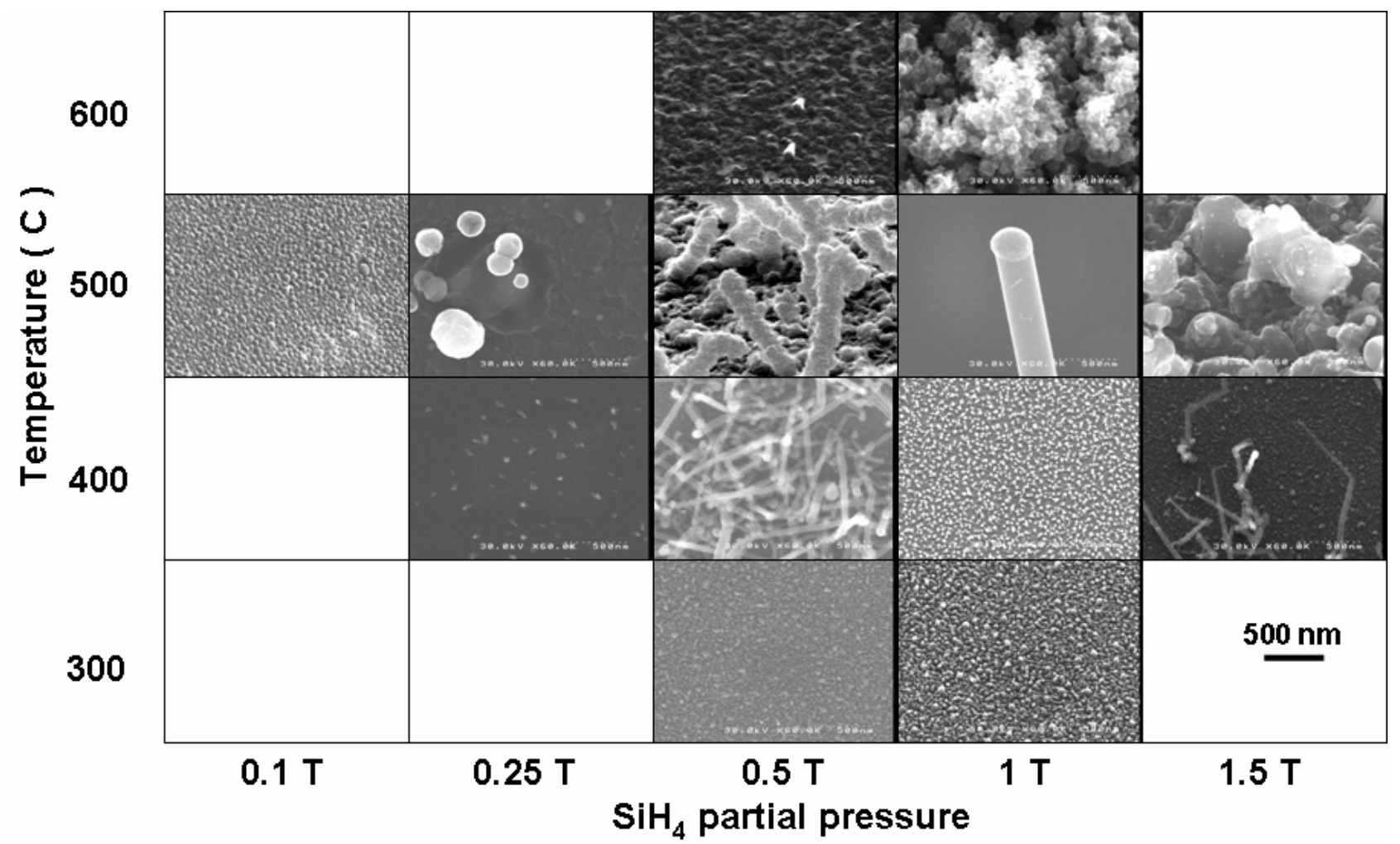

Fig. 1. Partial phase diagram of growth of silicon nanorods from In catalyst particles as a function of pressure and temperature. In all cases the growth was achieved with $\mathrm{SiH}_{4}$ diluted to $5 \%$ concentration in $\mathrm{Ar}$, with a flow rate of 100 sccm, for three hours. In most cases, catalyst particles were formed by the dewetting of a nominally $5 \mathrm{~nm}$ thick evaporated film of In, but the results of growth were found to be fairly relatively insensitive to catalyst layer thickness. 
leaving an array of catalyst particles embedded in holes in the oxide. This was then placed in the CVD reactor and VLS growth was performed under various conditions. As in the case of non-templated growth, optimal conditions were found to be different for the $\mathrm{Au}$ and In catalysts. Results of this process are displayed for In and Au in Figs. 3 (a) and 3 (b), respectively.

In addition to templating with electron-beam lithography, we have explored the use of porous alumina membranes in order to constrain the rod location and dimension, as well as reduce catalyst diffusion and evaporation. We used commercially available porous alumina membranes (Whatman Anopore ${ }^{\circledR}$ inorganic membranes), and evaporated $50 \mathrm{~nm}$ of catalyst (either In or $\mathrm{Au}$ ) on one side. The alumina was then coated with wax, and an approximately 12 um layer of nickel $(\mathrm{Ni})$ was then electrodeposited on the back side of the catalyst film. The wax was then removed and the alumina placed in the CVD chamber and deposition proceeded as before. Typical results from this process are shown in Fig. 4, after dissolution of the alumina membrane using sodium hydroxide. Note that because we use a solution-based approach to dissolving the alumina membranes, many of the silicon rods break during this process. We are currently working on alternative methods for dissolution of this membrane.

\section{Electrical Measurements}

Electrical measurements were performed on single nanorods by sonicating as-grown samples for $5-20$ s in 5-50 uL of IPA and dropcasting the resulting suspension in $5 \mathrm{uL}$ increments on $\mathrm{n}+, \sim 0.01 \mathrm{ohm} \mathrm{cm}$ Si wafers coated with $100 \mathrm{~nm}$ LPCVD silicon nitride $\left(\mathrm{Si}_{3} \mathrm{~N}_{4}\right)$, spinning the wafer at $\sim 300$ RPM for 1 minute between each dropcast. The nitride-coated wafers were then spincoated with a double layer of PMMA, and contacts were patterned in the PMMA by first locating a suitable rod (the rods were barely visible in the SEM through the PMMA), and then patterning a four-point probe assembly on top. After developing the pattern $5 \mathrm{~nm}$ of chromium $(\mathrm{Cr})$ and $1 \mathrm{um}$ of silver $(\mathrm{Ag})$ were evaporated and then lift-off was performed. Such a thick layer of Ag was used because the Si rods we were making contact to were so large in diameter that we needed a thick layer of metal in order to prevent the Si rods simply tearing through the metal contacts upon liftoff of the PMMA. We then wired-bonded these samples to 16-pin packages and performed two- and four-point I-V measurements as well as gate-bias-dependent two-point I-V measurements on the Si rods.

Rods characterized to date, grown from Au catalysts and $5 \% \mathrm{SiH}_{4}$ in $\mathrm{Ar}$ doped with 5 ppm phosphine, show resistivities of approximately $10^{-1} \mathrm{ohm} \mathrm{cm}$, indicating a dopant density of approximately $10^{17} \mathrm{~cm}^{-3}$, assuming n-type doping and the standard relationship between resistivity and dopant density for bulk silicon [14]. Backgated I-V measurements show no appreciable change in the conductivity of these nanorods for gate biases up to $50 \mathrm{~V}$, consistent with degenerate doping.

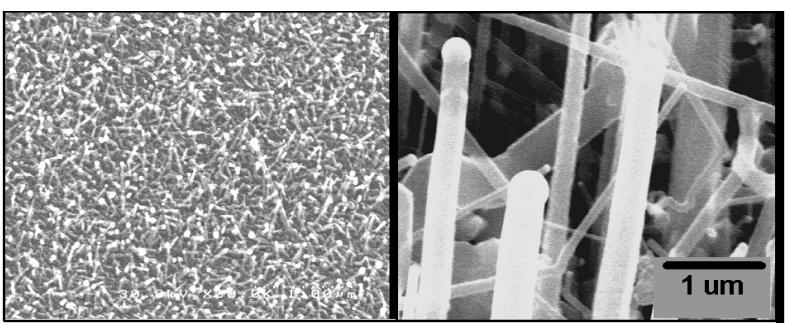

Fig. 2 (a)

Fig. 2 (b)

Typical results of CVD growth of silicon rods from nontemplated (a) indium and (b) gold catalyst. Both of these images are the result of introducing $5 \%$ silane in argon at a flow rate of $100 \mathrm{sccm}$ for three hours, with a $\mathrm{SiH} 4$ partial pressure of (a) 1 Torr and (b) 0.05 Torr, and substrate temperature of (a) $500 \mathrm{C}$ and (b) $600 \mathrm{C}$.

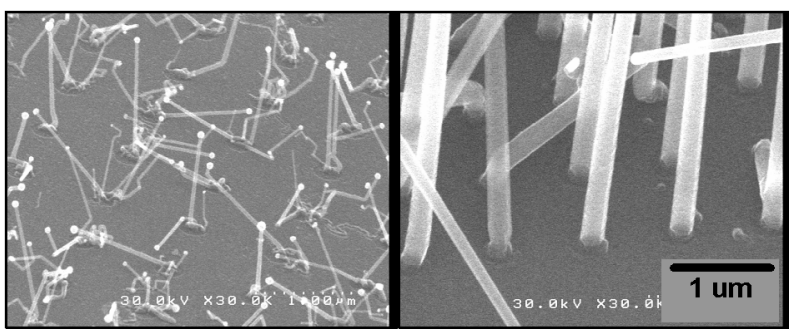

Fig. 3 (a)

Fig. 3 (b)

Typical results of CVD growth of Si rods, using electron beam lithography to pattern holes in an oxide on <111> silicon and then a lift-off step so that catalyst particles appear only in these holes, from (a) indium and (b) gold catalyst. These images are the result of introducing 5\% silane in argon at a flow rate of $100 \mathrm{sccm}$ for three hours, with a SiH4 partial pressure of (a) 1 Torr and (b) 0.05 Torr, and substrate temperature of (a) $500 \mathrm{C}$ and (b) $600 \mathrm{C}$ - hence the vastly different morphologies (see, for example, [6]). Nevertheless, these results demonstrate our control over the placement and size of the catalyst particles, and hence on the location of the onset of rod growth.

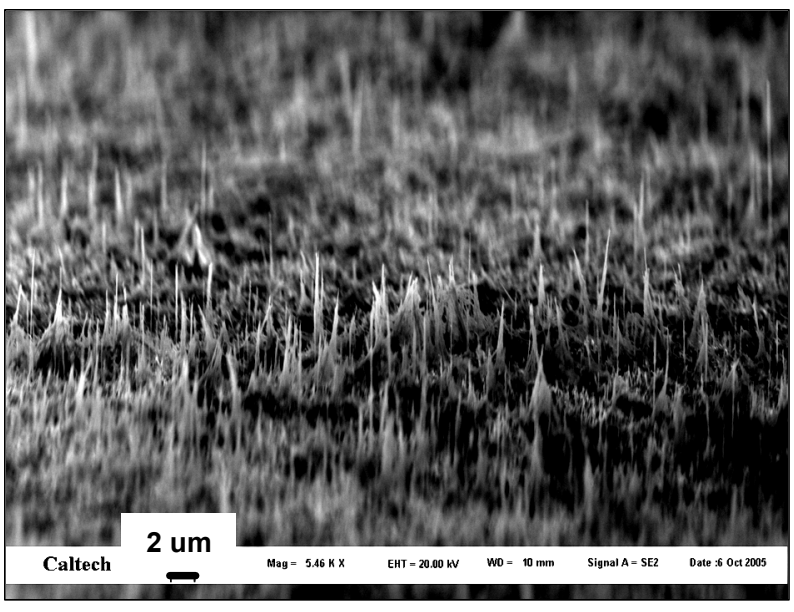

Fig. 4. Results from growth of Si nanorods from In catalyst within a porous alumina membrane, after dissolution of the alumina membrane using sodium hydroxide. 


\section{DISCUSSION AND FUTURE WORK}

The contrast between $\mathrm{Au}$ and In catalysts illustrate that it is possible in principle to form dense arrays of $\mathrm{Si}$ nanorods with diameters of $100 \mathrm{~s}$ of nms to several ums with or without templating (for the former catalyst), as well as form nanorods of these dimensions with a catalyst that does not form a deep-level trap state in the bandgap of Si (for the latter catalyst). To combine these properties is the ultimate aim of this work. Future work will involve exploring further the use of templating as a method to control rod growth from In catalysts, quantifying the differences in optoelectronic properties between rods grown with these two catalysts, demonstrating control over doping level within the rods, as well as a continued search for new VLS catalysts $\left(\mathrm{TiSi}_{2}\right.$ is another promising alternative to $\mathrm{Au}[15])$.

In order that nanowire and nanorod photovoltaic devices might make an impact in the solar cell industry, processing cost and robustness must be taken very seriously. For this reason, one would ultimately like to find processes that are not restricted by the high cost inherent in expensive growth catalysts such as In or $\mathrm{Au}$, or the extra processing steps inherent in the use and subsequent removal of a templating layer. Further, no discussion has yet been made in this paper about deposition of a top layer of opposite doping type to enable charge separation. This could be done in a variety of ways, this will be the subject of a future publication.

\section{CONCLUSIONS}

Nanorods are of interest in photovoltaic applications because of the potential for increased carrier collection in low diffusion length materials, and the potential for fabricating novel heterojunctions between non-latticematched materials. For a wholly inorganic, $p-n$ junction photovoltaic device design in $\mathrm{Si}$, rods with diameters of at least a few hundred $\mathrm{nm}$ so that the rod is able to sustain a depletion region, and lengths of tens of ums so that the rod absorbs most of the incident abovebandgap photons, are desired. Such rods have been fabricated by the VLS method with both Au and In as a growth catalyst. In has the advantage of not forming a deep-level trap state within Si's bandgap. However, without templating we have been unable to grow dense arrays of rods with In. Porous alumina and electronbeam lithography illustrate that such dense arrays can in principle be made with either catalyst. We have identified optimal growth conditions for both catalysts, with and without templates. Rods made from Au catalysts and phosphine doped silane displayed metal-like conductivity properties when electrical measurements on single wires were performed. Future work will include obtaining a better understanding and control of doping in the rods and how the choice of catalyst affects this.

\section{ACKNOWLEDGMENTS}

We would like to thank BP Solar, DOE, NSF, and Caltech's SURF program for financial support.

\section{REFERENCES}

[1] B.M. Kayes, N.S. Lewis, and H.A. Atwater, "Comparison of the device physics principles of planar and radial p-n junction nanorod solar cells", Jour. App. Phys., 97, 2005, Art. No. 114302.

[2] B. Kannan, K. Castelino, and A. Majumdar, "Design of nanostructured heterojunction polymer photovoltaic de-vices", Nano Letters, 3, 2003, pp. 1729-1733.

[3] M.S. Gudiksen et al., "Growth of nanowire superlattice structures for nanoscale photonics and electronics", Nature, 415, 2002, pp. 617-620.

[4] W.U. Huynh, J.J Dittmer, and A.P. Alivisatos, "Hybrid nanorod-polymer solar cells", Science, 295, 2002 pp. 2425-2427.

[5] N.A. Melosh et al., "Ultrahigh-density nanowire lattices and circuits", Science, 300, 2003, pp. 112-115.

[6] J. Westwater, D.P. Gosain, and S. Usui, "Control of the Size and Position of Silicon Nanowires Grown via the Vapor-Liquid-Solid Technique", Jour. Vac. Sci. \& Tech. B, 15, 1997, pp. 554-557.

[7] B.M. Kayes et al., "Radial PN Junction Nanorod Solar Cells: Device Physics Principles and Routes to Fabrication in Silicon“, IEEE PVSC Proceedings, 2005.

[8] A.L. Fahrenbruch, and R.H. Bube, Fundamentals of Solar Cells: Photovoltaic Solar Energy Conversion, Academic Press Ltd., Stanford, 1983, p. 255.

[9] S.M. Sze, Physics of Semiconductor Devices, WileyInterscience, New York, 1969, pp. 21, 37, and 850.

[10] T. B. Massalski (ed.), Binary alloy phase diagrams, ASM International, Ohio, 1990, p. 428.

[11] Y. Civale et al., "Aspects of Silicon Nanowire Synthesis by Aluminum-Catalyzed Vapor-Liquid-Solid Mechanism", http://med.tn.tudelft.nl/ hadley/ publications/safe/civale-safe2004.pdf

[12] V.A. Nebol'sin and A.A. Shchetinin, "Role of Surface Energy in the Vapor-Liquid-Solid Growth of Silicon", Inorganic Materials 39, 2003, pp. 899-903.

[13] http://www.veeco.com/learning/ learning vaporelements.asp

[14] http://www.ioffe.rssi.ru/SVA/NSM/Semicond/Si/ Figs/135.gif

[15] T.I. Kamins et al., "Ti-catalyzed Si nanowires by chemical vapor deposition: Microscopy and growth mechanisms“, Jour. App. Phys., 89, 2001, pp. 10081016. 